Moscow Clinical Scientific Centre n.a. A.S. Loginov, Moscow, Russia

\title{
Audit of using El-Ganzouri prognosis scale for difficult airway assessment in obese patients.
}

\author{
Malakhova A., Klimov A., Kamnev S., Subbotin V.
}

\section{Background:}

Difficult laryngoscopy and intubation in obesity patients is more frequent than in patients with normal BMI $[1,2,3,4]$. Adequate assessment of difficult airway before surgery with general anaesthesia is topical. The aim of our study was to audit predictive value of El-Ganzouri scale for patients with BMI more than $30 \mathrm{~kg} / \mathrm{m}^{2}$.

Materials and methods:

Our Center has accepted management protocol for patients with BMI more than $30 \mathrm{Kg} / \mathrm{m} 2$ since 2016. This protocol includes assessment of airway by El-Ganzouri scale and intubation by videolaryngoscope in all cases. ElGanzouri scale included 7 measures: Mallampati class, Body weight, Anamnesis of difficult intubation.

Mouth open

$>4 \mathrm{~cm}-0$ point

$<4 \mathrm{~cm}-1$ point

Thiromental

distance

$>6,5 \mathrm{~cm}-$

$<6,0 \mathrm{~cm}-\quad 2$ point

The possibility of mandible extension Yes - 0 point No - 1 point

Neck mobility $>90^{\circ}-0$ point $80-90^{\circ}-1$ point $<80^{\circ}-2$ point

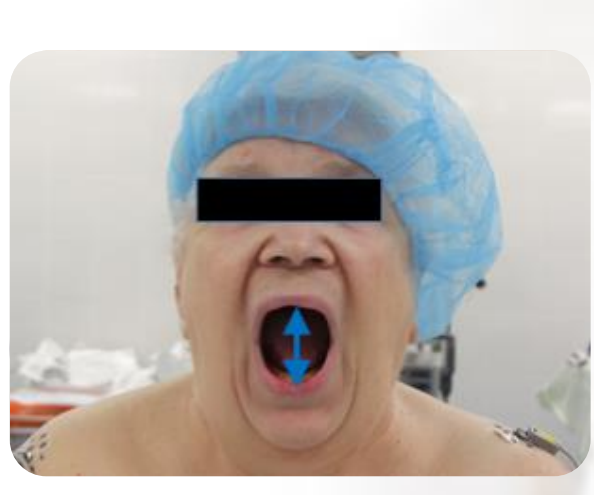

\section{Selection device}
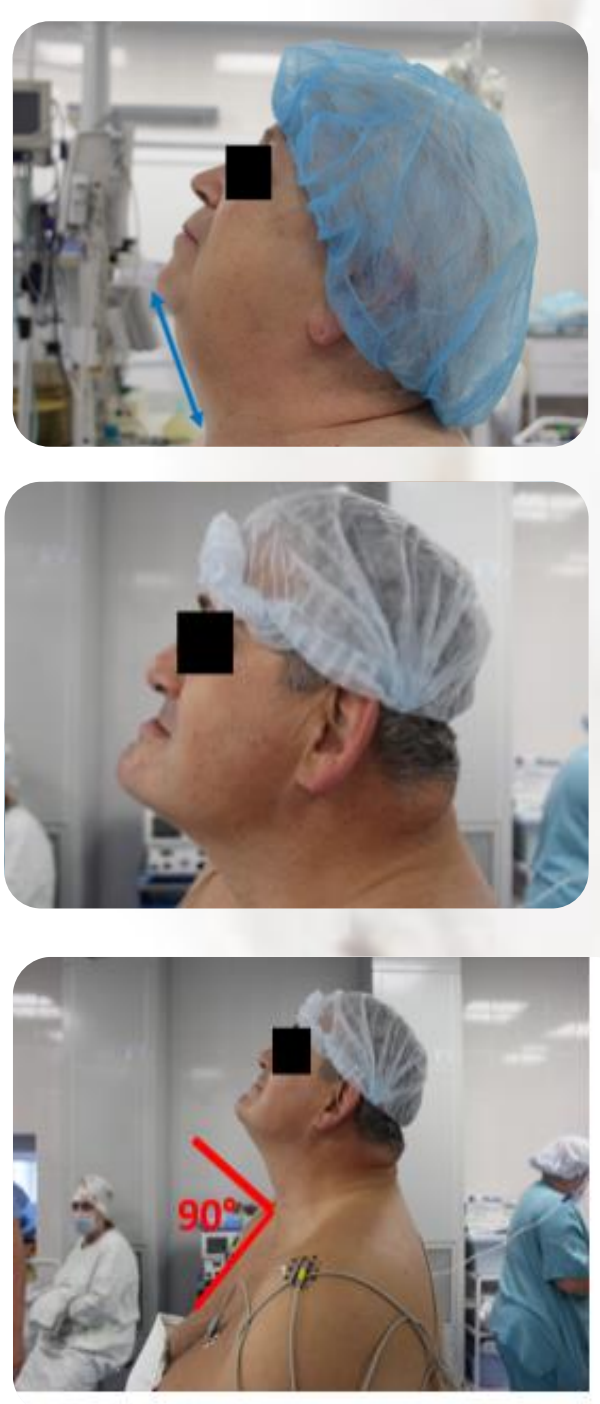

0 till 3 points

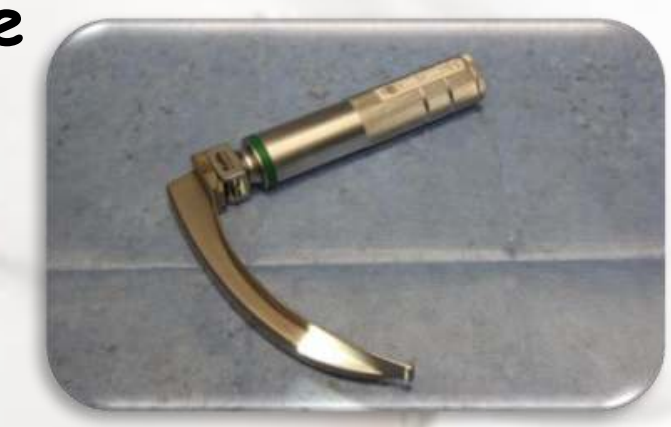

4 till 7 points

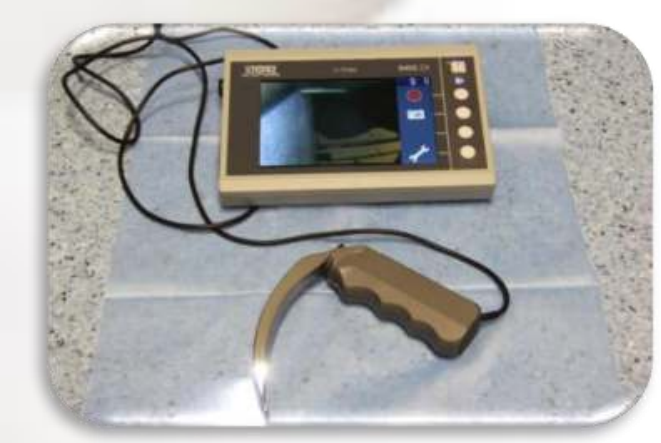

$\geq 8$ points

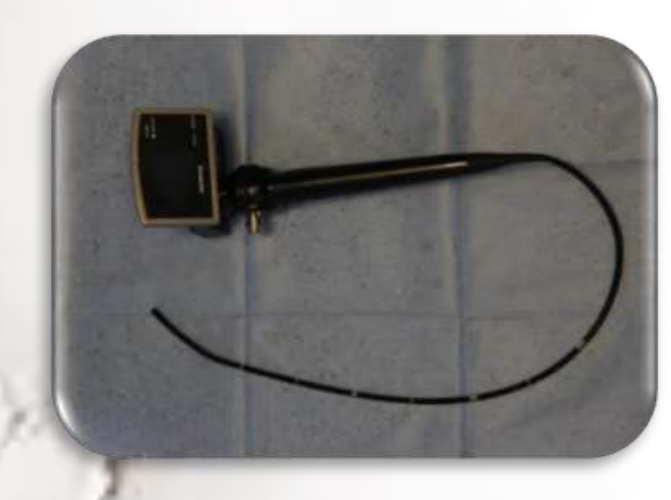

During our audit were assessed 50 patients (29 women and 21 men, aged from 26 to 74 ) which have general anaesthesia with tracheal intubation for elective bariatric surgery.
On the first step anaesthesiologysts try to intubate the patients was using videolaryngoscope as standard laryngoscope. If it was not possible anaesthesiologysts used this device as videolaryngoscope.

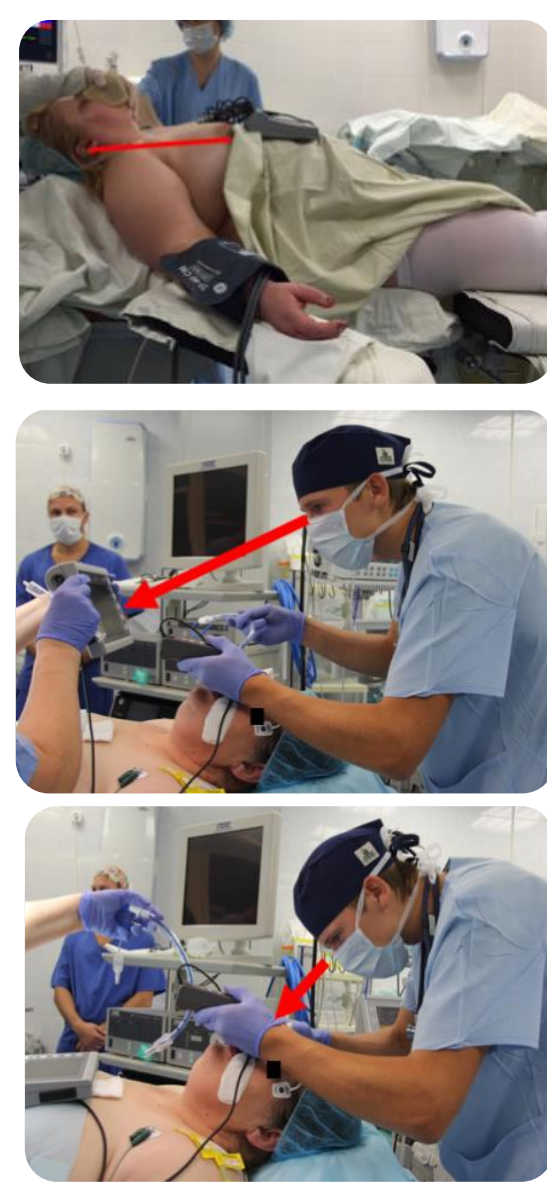

Results:

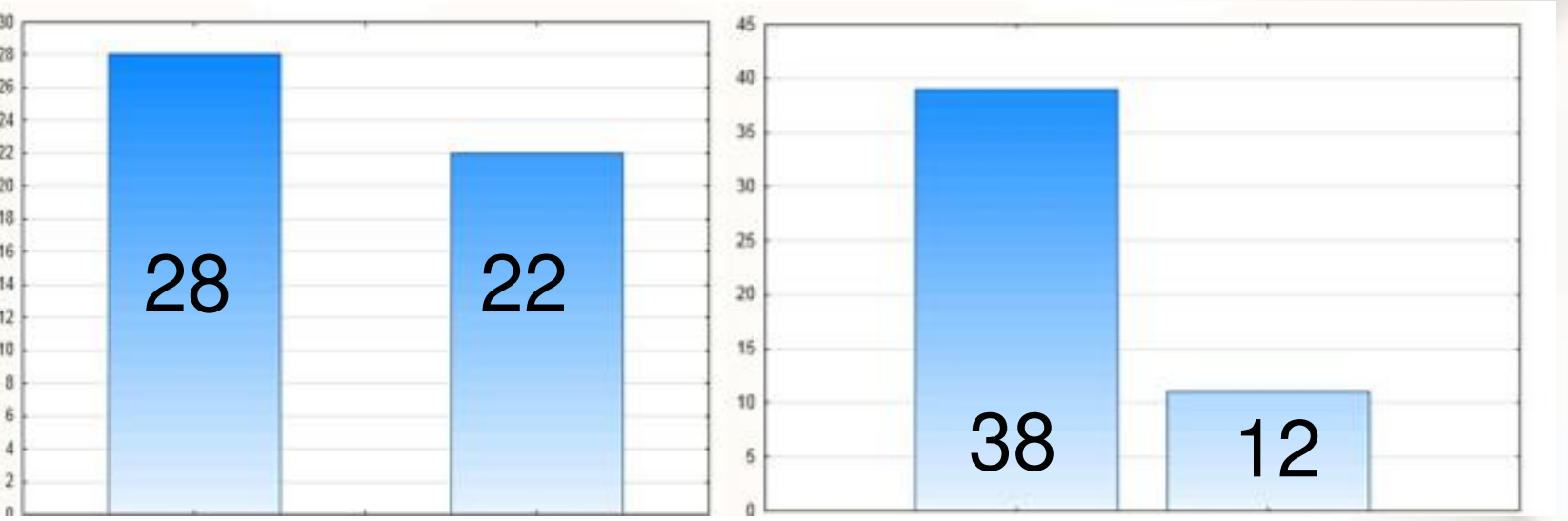

Results of intubation sensivity- $81 \%$, specificy- $66 \%$,

p. predictability $-40 \%$,

n. predictability- $93 \%$.

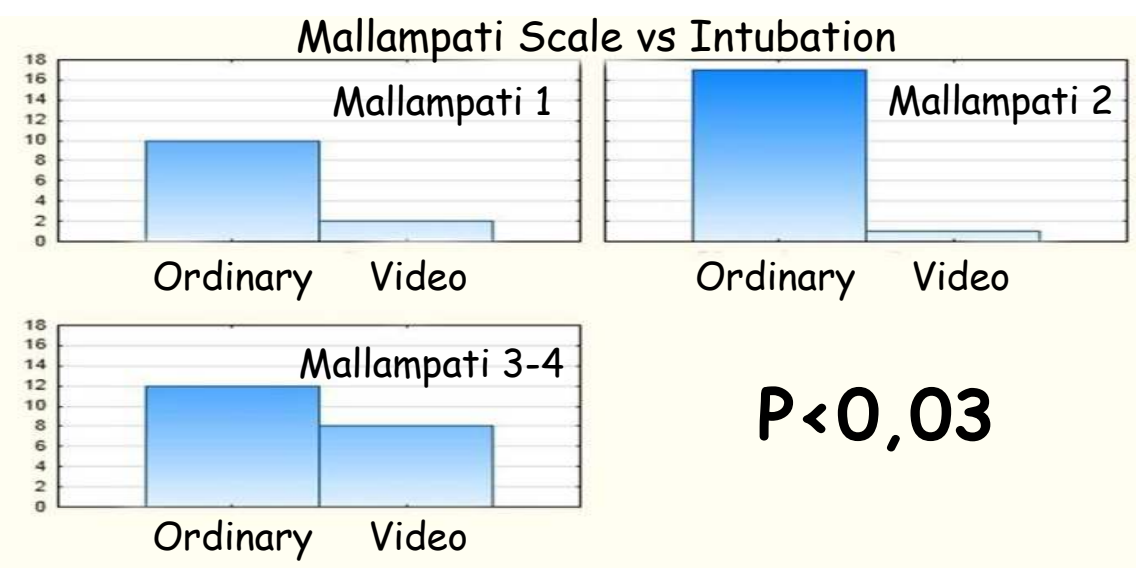

\section{Conclusion:}

We recommend El-Ganzouri scale for airway assessment in obese patients. This scale has high prognosis value for standard laryngoscopy. If El-Ganzouri scale recommends using video laryngoscope, it is better to use it even if intubation may be successful with a standard laryngoscope. However, assessment score of difficult airway in obese patients requires further study. 